AJMS

27,1

2

Received 28 February 2019 Accepted 29 June 2019

\section{On warped product bi-slant submanifolds of Kenmotsu manifolds}

\author{
Siraj Uddin \\ Department of Mathematics, Faculty of Science, King Abdulaziz University, \\ Jeddah, Saudi Arabia \\ Ion Mihai \\ Faculty of Mathematics and Computer Science, University of Bucharest, \\ Bucharest, Romania, and \\ Adela Mihai \\ Department of Mathematics and Computer Science, \\ Technical University of Civil Engineering Bucharest, Bucharest, Romania
}

\begin{abstract}
Chen (2001) initiated the study of CR-warped product submanifolds in Kaehler manifolds and established a general inequality between an intrinsic invariant (the warping function) and an extrinsic invariant (second fundamental form).

In this paper, we establish a relationship for the squared norm of the second fundamental form (an extrinsic invariant) of warped product bi-slant submanifolds of Kenmotsu manifolds in terms of the warping function (an intrinsic invariant) and bi-slant angles. The equality case is also considered. Some applications of derived inequality are given.
\end{abstract}

Keywords Warped products, Semi-slant, Pseudo-slant, Bi-slant submanifolds, Warped product bi-slant submanifolds, Kenmotsu manifolds

Paper type Original Article

\section{Introduction}

In [18], K. Kenmotsu studied one class of almost contact metric manifolds known as Kenmotsu manifolds. He proved that:

\section{JEL Classification - 53C15, 53C40, 53C42, 53B25}

(C) Siraj Uddin, Ion Mihai and Adela Mihai. Published in the Arab Journal of Mathematical Sciences. Published by Emerald Publishing Limited. This article is published under the Creative Commons Attribution (CCBY 4.0) license. Anyone may reproduce, distribute, translate and create derivative works of this article (for both commercial and non-commercial purposes), subject to full attribution to the original publication and authors. The full terms of this license may be seen at http://creativecommons. org/licences/by/4.0/legalcode

Declaration of Competing Interest: The authors declare that they have no known competing financial interests or personal relationships that could have appeared to influence the work reported in this paper.

The publisher wishes to inform readers that the article "On warped product bi-slant submanifolds of Kenmotsu manifolds" was originally published by the previous publisher of the Arab Journal of Mathematical Sciences and the pagination of this article has been subsequently changed. There has been no change to the content of the article. This change was necessary for the journal to transition from the previous publisher to the new one. The publisher sincerely apologises for any inconvenience caused. To access and cite this article, please use Uddin, S., Mihai, I., Mihai, A. (2019), "On warped product bi-slant submanifolds of Kenmotsu manifolds”, Arab Journal of Mathematical Sciences, Vol. 27 No. 1, pp. 2-14. The original publication date for this paper was 05/07/2019. 
1. Locally a Kenmotsu manifold is a warped product $I \times{ }_{f} M$ of an interval $I$ and a Kaehler manifold $M$, with warping function $f=c e^{t}$, where $c$ is a nonzero constant.

2. A Kenmotsu manifold with constant sectional curvature is a space of constant curvature -1 , and so it is locally a hyperbolic space.

A $(2 m+1)$-dimensional manifold $\tilde{M}$ is said to be an almost contact manifold if it admits an endomorphism $\varphi$ of its tangent bundle $T \tilde{M}$, a vector field $\xi$ and a 1-form $\eta$, which satisfy:

$$
\varphi^{2}=-I+\eta \otimes \xi, \varphi \xi=0, \eta(\xi)=0, \eta \circ \varphi=0 .
$$

There exists a compatible metric $g$, which satisfies

$$
g(\varphi X, \varphi Y)=g(X, Y)-\eta(X) \eta(Y), \eta(X)=g(X, \xi),
$$

for all vector fields $X, Y$ on $\tilde{M}$ [6]. In addition, an almost contact metric manifold $\tilde{M}$ is said to be a Kenmotsu manifold [18] if the relation

$$
\left(\tilde{\nabla}_{X} \varphi\right) Y=g(\varphi X, Y) \xi-\eta(Y) \varphi X
$$

holds, where $\tilde{\nabla}$ is the Levi-Civita connection of $g$. From (1.3), for a Kenmotsu manifold $\tilde{M}$, we also have

$$
\tilde{\nabla}_{X} \xi=X-\eta(X) \xi
$$

As Kenmotsu manifolds are warped product manifolds, therefore it is interesting to investigate the geometry of its warped product submanifolds. The notion of warped submanifolds was first introduced by B.-Y. Chen as a CR-warped product submanifold of Kaehler manifolds in his series of articles [11,12]. He established a general sharp inequality between the main extrinsic invariant (the second fundamental form) and an intrinsic invariant (the warping function) of such submanifolds. Motivated by Chen's work many geometers studied warped product submanifolds for different spaces (see, e.g., [4,17,19-25,29,30] among many others. For the most up-to-date overview of this subject, see [13-15]).

On the other hand, J.L. Cabrerizro et al. studied in [7] bi-slant submanifolds of almost contact metric manifolds. In [28], the first author and B.-Y. Chen investigated warped product bi-slant submanifolds in Kaehler manifolds. They proved that there do not exist any warped product bi-slant submanifolds of Kaehler manifolds other than hemi-slant warped products and CR-warped products. The non-existence of warped product bi-slant submanifolds is proved in [2] for cosymplectic manifolds.

In this paper, we study warped product bi-slant submanifolds of a Kenmotsu manifold. The geometry of such submanifolds in Kenmotsu manifolds is quite different from Sasakian and cosymplectic case because in case of Kenmotsu manifolds such submanifolds exist while there is no proper warped product bi-slant submanifolds in Sasakain and cosymplectic as well. On their existence, we establish a generalized Chen type sharp inequality for the squared norm of the second fundamental form in terms of the warping function and bi-slant angles. The equality case is considered. Some applications are given in the last section.

\section{Preliminaries}

Let $\psi: M^{n} \rightarrow M^{(n+d)}$ be an isometric immersion of an $n$-dimensional Riemannian manifold $M$ into an $(n+d)$-dimensional Riemannian manifold $\tilde{M}$. We denote by $\nabla$ and $\tilde{\nabla}$ the LeviCivita connections on $M$ and $\tilde{M}$, respectively. Then the Gauss and Weingarten formulas are respectively given by [31]

$$
\tilde{\nabla}_{X} Y=\nabla_{X} Y+h(X, Y)
$$

Submanifolds of Kenmotsu manifolds 
AJMS

27,1

and

$$
\tilde{\nabla}_{X} V=-A_{V} X+\nabla_{X}^{\perp} V
$$

for any $X, Y \in T M$ and $V \in T^{\perp} M$, where $\nabla^{\perp}$ is the normal connection in the normal bundle $T^{\perp} M$ and $A_{V}$ is the shape operator of $M$ with respect to $V$. Moreover, $h: T M \times T M \rightarrow T^{\perp} M$ is the second fundamental form of $M$ in $\tilde{M}$. Furthermore, $A_{V}$ and $h$ are related by

$$
g(h(X, Y), V)=g\left(A_{V} X, Y\right),
$$

for any $X, Y \in T M$ and $V \in T^{\perp} M$.

A submanifold $M$ of a Riemannian manifold $\tilde{M}$ is said to be a totally umbilical submanifold if $h(X, Y)=g(X, Y) H$, for any $X, Y \in T M$, where $H=\frac{1}{n} \sum_{i=1}^{n} h\left(e_{i}, e_{i}\right)$ is the mean curvature vector of $M$. A submanifold $M$ is said to be totally geodesic if $h(X, Y)=0$. Also, one denotes

$$
\|h\|^{2}=\sum_{i, j=1}^{n} g\left(h\left(e_{i}, e_{j}\right), h\left(e_{i}, e_{j}\right)\right), \quad h_{i j}^{r}=g\left(h\left(e_{i}, e_{j}\right), e_{r}\right),
$$

with $i, j=1, \ldots, n ; r=n+1, \ldots, n+d$, where $\left\{e_{1}, \ldots, e_{n}\right\}$ is an orthonormal basis of the tangent space $T_{p} M$ and $\left\{e_{n+1}, \ldots, e_{n+d}\right\}$ an orthonormal basis of the normal space $T_{p}^{\perp}$, for any $p \in M$.

For a differentiable function $f$ on a $m$-dimensional manifold $\tilde{M}$, the gradient $\vec{\nabla} f$ of $f$ is defined as $g(\vec{\nabla} f, X)=X(f)$, for any $X$ tangent to $\tilde{M}$.

For any vector field $X$ tangent to $M$, we write

$$
\varphi X=T X+F X
$$

where $T X$ is the tangential component and $F X$ is the normal component of $\varphi X$. Thus, $T$ is an endomorphism on the tangent bundle $T M$ and $F$ is a normal bundle valued 1-form of $T M$. A submanifold $M$ is called invariant if $F$ is identically zero, that is, $\varphi X \in T M$ for any $X \in T M$; while, $M$ is anti-invariant if $T$ is identically zero, that is, $\varphi X \in T^{\perp} M$, for any $X \in T M$.

Similarly, for any vector field $V$ normal to $M$, we put

$$
\varphi V=t V+f V
$$

where $t V$ and $f V$ are the tangential and normal components of $\varphi V$, respectively.

Let $M$ be a submanifold of an almost contact metric manifold $(\tilde{M}, \varphi, \xi, \eta, g)$. Hence, if we denote $\mathfrak{D}$ the orthogonal distribution to $\xi$ in $T M$, then $T M=\mathfrak{D} \oplus\langle\xi\rangle$, where $\langle\xi\rangle$ is the 1-dimensional distribution spanned by $\xi$. For any nonzero vector $X$ tangent to $M$ at the point $p \in M$, such that $X$ is not proportional to $\xi_{p}$, we denote by $\theta(X)$, the angle between $\varphi X$ and $T_{p} M$. In fact, since $\varphi \xi=0, \theta(X)$ agrees with the angle between $\varphi X$ and $\mathfrak{D}_{p}$. A submanifold $M$ of an almost contact metric manifold $\tilde{M}$ is said to be slant [8], if for any non-zero vector $X$ tangent to $M$ at $p$ such that $X$ is not proportional to $\xi_{p}$, the angle $\theta(X)$ between $\varphi X$ and $T_{p} M$ is constant, i.e., it does not depend on the choice of $p \in M$ and $X \in T_{p} M-\left\langle\xi_{p}\right\rangle$. In this case $D$ is a slant distribution with slant angle $\theta$.

A slant submanifold is said to be proper slant, if neither $\theta=0$ nor $\theta=\frac{\pi}{2}[9,10]$. We note that on a slant submanifold if $\theta=0$, then it is an invariant submanifold and if $\theta=\frac{\pi}{2}$, then it is an anti-invariant submanifold. A slant submanifold is said to be proper slant if it is neither invariant nor anti-invariant.

A characterization of slant submanifolds was given in [8] as follows:

Theorem 1 ([8]). Let $M$ be a submanifold of an almost contact metric manifold $\tilde{M}$ such that $\xi \in T M$. Then $M$ is slant if and only if there exists a constant $\lambda \in[0,1]$ such that 


$$
T^{2}=\lambda(-I+\eta \otimes \xi) .
$$

Furthermore, in such case, if $\theta$ is slant angle, then $\lambda=\cos ^{2} \theta$.

The following relations are straightforward consequences of (2.7)

$$
\begin{gathered}
g(T X, T Y)=\cos ^{2} \theta[g(X, Y)-\eta(X) \eta(Y)], \\
g(F X, F Y)=\sin ^{2} \theta[g(X, Y)-\eta(X) \eta(Y)],
\end{gathered}
$$

for any $X, Y$ tangent to $M$.

The following useful relation is obtained as a consequence of (2.7) in [26].

Theorem 2 ([26]). Let $M$ be a proper slant submanifold of an almost contact metric manifold M. Then

$$
\text { (a) } t F X=\sin ^{2} \theta(-X+\eta(X) \xi), \quad(b) \quad f F X=-F T X,
$$

for any $X \in T M$.

Another characterization of slant submanifolds was given in [7]:

Theorem 3 ([7]). Let $D$ be a distribution on $M$, orthogonal to $\xi$. Then, D is slant if and only if there exists a constant $\lambda \in[0,1]$ such that $(P T)^{2} X=-\lambda X$, for any $X \in \mathfrak{D}_{p}$ at $p \in M$, where $P$ denotes the orthogonal projection on $\mathfrak{D}$. Furthermore, in this case, $\lambda=\cos ^{2} \theta_{\mathfrak{D}}$.

J.L. Cabrerizo et al. [7] defined bi-slant submanifolds as follows:

Definition 1. A submanifold $M$ of an almost contact metric manifold $\tilde{M}$ is said to be bi-slant if there exists a pair of orthogonal distributions $\mathfrak{D}_{1}$ and $\mathfrak{D}_{2}$ on $M$ such that:

(i) The tangent bundle $T M$ admits the orthogonal direct decomposition: $T M=$ $\mathfrak{D}_{1} \oplus \mathfrak{D}_{2} \oplus\langle\xi\rangle$.

(ii) Each $\mathfrak{D}_{i} \forall i=1,2$ is a slant distribution with slant angle $\theta_{i}$.

Given a bi-slant submanifold $M$, for any $X \in T M$, we put

$$
X=P_{1} X+P_{2} X+\eta(X) \xi,
$$

where $P_{i} X$ denotes the component of $X$ in $\mathfrak{D}_{i}$, for any $i=1,2$. In particular, if $X \in \mathfrak{D}_{i}$, then we obtain $X=P_{i} X$. If we define $T_{i}=P_{i} \circ T$, then we have

$$
\varphi X=T_{1} X+T_{2} X+F X,
$$

for any $X \in T M$. Given $i=1$, 2, from Theorem 3, we get

$$
T_{i}^{2} X=-\cos ^{2} \theta_{i} X
$$

for any $X \in \mathfrak{D}_{i}$.

Non-trivial examples of bi-slant submanifolds are given in [7].

\section{Warped product bi-slant submanifolds}

Let $M_{1} \times{ }_{f} M_{2}$ be a warped product manifold of two Riemannian manifolds $M_{1}$ and $M_{2}$. Then from a result of [5], we have

$$
\nabla_{X} Z=\nabla_{Z} X=\frac{1}{f}(X f) Z
$$

for any vector fields $X, Z$ tangent to $M_{1}, M_{2}$, respectively. 
AJMS

27,1

6

Recently, B.-Y. Chen and the first author introduced the notion of warped product bi-slant submanifolds of Kaehler manifolds [28]. They proved the non-existence of proper warped product bi-slant submanifolds. Then, they introduced the notion of warped product pointwise bi-slant submanifolds and obtained several fundamental results [16]. In this section, we give some useful lemmas for warped product bi-slant submanifolds of Kenmotsu manifolds. First we define these submanifolds as follows:

A warped product $M_{1} \times{ }_{f} M_{2}$ of two slant submanifolds $M_{1}$ and $M_{2}$ with slant angles $\theta_{1}$ and $\theta_{2}$ of a Kenmotsu manifold $\tilde{M}$ is called a warped product bi-slant submanifold.

A warped product bi-slant submanifold $M=M_{1} \times{ }_{f} M_{2}$ is called proper if both $M_{1}$ and $M_{2}$ are proper slant submanifolds with slant angles $\theta_{1}, \theta_{2} \neq 0, \frac{\pi}{2}$ of $\tilde{M}$. A warped product bislant submanifold $M_{1} \times{ }_{f} M_{2}$ is a contact CR-warped product if $\theta_{1}=0, \theta_{2}=\frac{\pi}{2}$ or $\theta_{2}=0, \theta_{1}=\frac{\pi}{2}$; such submanifolds were discussed in [3,27]. Also, it is a warped product pseudo-slant submanifold if $\theta_{1}=\theta$ and $\theta_{2}=\frac{\pi}{2}$ [1] or $\theta_{2}=\theta$ and $\theta_{1}=\frac{\pi}{2}$ [23]. The warped product bi-slant submanifolds with slant angles $\theta_{1}=0, \theta_{2}=\theta$ or $\theta_{2}=0, \theta_{1}=\theta$ were discussed in [25,30].

Let $M=M_{1} \times{ }_{f} M_{2}$ be a warped product bi-slant submanifold of a Kenmotsu manifold $\tilde{M}$ such that the structure vector field $\xi$ is tangent to $M$, where $M_{1}$ and $M_{2}$ are proper slant submanifolds of $\tilde{M}$. Then, we distinguish 2 cases:

(i) $\xi$ is tangent to $M_{1}$;

(ii) $\xi$ is tangent to $M_{2}$.

From (1.4), (2.1) and (3.1), the second case is trivial i.e., there does not exist any proper warped product bi-slant submanifold of a Kenmotsu manifold when the structure vector field is tangent to the fiber.

Now, we start with the case (i). Throughout this paper, we assume that the tangent spaces of $M_{1}$ and $M_{2}$, respectively are $\mathfrak{D}_{1}$ and $\mathfrak{D}_{2}$. From now on, we use the following conventions: $X_{1}, Y_{1}$ are vector fields in $\mathfrak{D}_{1}$ and $X_{2}, Y_{2}$ are vector fields in $\mathfrak{D}_{2}$.

Lemma 1. Let $M=M_{1} \times{ }_{f} M_{2}$ be a warped product bi-slant submanifold of a Kenmotsu manifold $\tilde{M}$ such that $\xi \in \mathfrak{D}_{1}$. Then, we have

(i) $\xi(\ln f)=1$;

(ii) $g\left(h\left(X_{1}, Y_{1}\right), F X_{2}\right)=g\left(h\left(X_{1}, X_{2}\right), F Y_{1}\right)$,

for any $X_{1}, Y_{1} \in \mathfrak{D}_{1}$ and $X_{2} \in \mathfrak{D}_{2}$.

Proof. First part is trivial and can be obtained by using (1.4), (2.1) and (3.1). For the second part, we have

$$
g\left(\tilde{\nabla}_{X_{1}} Y_{1}, X_{2}\right)=-g\left(\nabla_{X_{1}} X_{2}, Y_{1}\right)=-X_{1}(\ln f) g\left(Y_{1}, X_{2}\right)=0,
$$

for any $X_{1}, Y_{1} \in \mathfrak{D}_{1}$ and $X_{2} \in \mathfrak{D}_{2}$. Also, from (1.2) and the fact that $\xi$ is tangent to $M_{1}$, we have

$$
g\left(\tilde{\nabla}_{X_{1}} Y_{1}, X_{2}\right)=g\left(\varphi \tilde{\nabla}_{X_{1}} Y_{1}, \varphi X_{2}\right)=g\left(\tilde{\nabla}_{X_{1}} \varphi Y_{1}, \varphi X_{2}\right)-g\left(\left(\tilde{\nabla}_{X_{1}} \varphi\right) Y_{1}, \varphi X_{2}\right) .
$$

Form (3.2) and (3.3), we obtain

$$
\begin{aligned}
g\left(\left(\tilde{\nabla}_{X_{1}} \varphi\right) Y_{1}, \varphi X_{2}\right)= & g\left(\tilde{\nabla}_{X_{1}} \varphi Y_{1}, \varphi X_{2}\right)=g\left(\tilde{\nabla}_{X_{1}} T_{1} Y_{1}, T_{2} X_{2}\right) \\
& +g\left(\tilde{\nabla}_{X_{1}} T_{1} Y_{1}, F X_{2}\right)+g\left(\tilde{\nabla}_{X_{1}} F Y_{1}, \varphi X_{2}\right) .
\end{aligned}
$$

Then using the covariant derivative property of Riemannian connection and (1.2), (1.3), (2.1) and (3.1), we derive 


$$
\begin{aligned}
\eta\left(Y_{1}\right) g\left(T_{1} X_{1}, T_{2} X_{2}\right)= & -X_{1}(\ln f) g\left(T_{1} Y_{1}, T_{2} X_{2}\right)+g\left(h\left(X_{1}, T_{1} Y_{1}\right), F X_{2}\right) \\
& +g\left(\left(\tilde{\nabla}_{X_{1}} \varphi\right) F Y_{1}, X_{2}\right)-g\left(\tilde{\nabla}_{X_{1}} \varphi F Y_{1}, X_{2}\right) .
\end{aligned}
$$

By the orthogonality of vector fields, the left hand side and the first term in the right hand side vanish identically. Then using (1.3), (2.6) and (2.10), we find

$$
\begin{aligned}
0=g\left(h\left(X_{1}, T_{1} Y_{1}\right), F X_{2}\right) & +\sin ^{2} \theta_{1} g\left(\tilde{\nabla}_{X_{1}} Y_{1}, X_{2}\right)-\sin ^{2} \theta_{1} g\left(\tilde{\nabla}_{X_{1}} \xi, X_{2}\right) \\
& +g\left(\tilde{\nabla}_{X_{1}} F T_{1} Y_{1}, X_{2}\right) .
\end{aligned}
$$

Using (1.4), (2.2), (2.3), (3.1) and the orthogonality of vector fields, we derive

$$
g\left(h\left(X_{1}, X_{2}\right), F T_{1} Y_{1}\right)=g\left(h\left(X_{1}, T_{1} Y_{1}\right), F X_{2}\right) .
$$

Interchanging $Y_{1}$ by $T_{1} Y_{1}$ in (3.4), we obtain

$$
\begin{aligned}
\cos ^{2} \theta_{1} g\left(h\left(X_{1}, X_{2}\right), F Y_{1}\right) & =\cos ^{2} \theta_{1} g\left(h\left(X_{1}, Y_{1}\right), F X_{2}\right) \\
& -\cos ^{2} \theta_{1} \eta\left(Y_{1}\right) g\left(h\left(X_{1}, \xi\right), X_{2}\right) .
\end{aligned}
$$

Since for a submanifold $M$ of a Kenmotsu manifold $\tilde{M}, h(X, \xi)=0, \forall X \in T M$, then the second part of the lemma follows from above relation. Hence, the proof is complete.

Lemma 2. Let $M=M_{1} \times_{f} M_{2}$ be a warped product bi-slant submanifold of a Kenmotsu manifold $\tilde{M}$ such that $\xi \in \mathfrak{D}_{1}$, where $M_{1}$ and $M_{2}$ are proper slant submanifolds of $\tilde{M}$ with slant angles $\theta_{1}$ and $\theta_{2}$, respectively. Then, we have

$$
\begin{aligned}
g\left(h\left(X_{2}, Y_{2}\right), F T_{1} X_{1}\right)-g\left(h\left(X_{2}, T_{1} X_{1}\right), F Y_{2}\right)= & T_{1} X_{1}(\ln f) g\left(X_{2}, T_{2} Y_{2}\right) \\
& -\cos ^{2} \theta_{1}\left(X_{1}(\ln f)-\eta\left(X_{1}\right)\right) g\left(X_{2}, Y_{2}\right)
\end{aligned}
$$

for any $X_{1} \in \mathfrak{D}_{1}$ and $X_{2}, Y_{2} \in \mathfrak{D}_{2}$.

Proof. For any $X_{1} \in \mathfrak{D}_{1}$ and $X_{2}, Y_{2} \in \mathfrak{D}_{2}$, we have

$$
g\left(\tilde{\nabla}_{X_{2}} X_{1}, Y_{2}\right)=X_{1}(\ln f) g\left(X_{2}, Y_{2}\right) .
$$

On the other hand, we also have

$$
g\left(\tilde{\nabla}_{X_{2}} X_{1}, Y_{2}\right)=g\left(\varphi \tilde{\nabla}_{X_{2}} X_{1}, \varphi Y_{2}\right)=g\left(\tilde{\nabla}_{X_{2}} \varphi X_{1}, \varphi Y_{2}\right)-g\left(\left(\tilde{\nabla}_{X_{2}} \varphi\right) X_{1}, \varphi Y_{2}\right) .
$$

Using (1.3) and (2.5), we arrive at

$$
\begin{aligned}
g\left(\tilde{\nabla}_{X_{2}} X_{1}, Y_{2}\right)= & g\left(\tilde{\nabla}_{X_{2}} T_{1} X_{1}, T_{2} Y_{2}\right)+g\left(\tilde{\nabla}_{X_{2}} T_{1} X_{1}, F Y_{2}\right) \\
& +g\left(\tilde{\nabla}_{X_{2}} F X_{1}, \varphi Y_{2}\right)+\eta\left(X_{1}\right) g\left(\varphi X_{2}, \varphi Y_{2}\right) .
\end{aligned}
$$

From (1.2), (2.1) and (3.1), the above equation takes the form

$$
\begin{aligned}
g\left(\tilde{\nabla}_{X_{2}} X_{1}, Y_{2}\right)= & T_{1} X_{1}(\ln f) g\left(X_{2}, T_{2} Y_{2}\right)+g\left(h\left(X_{2}, T_{1} X_{1}\right), F Y_{2}\right)-g\left(\tilde{\nabla}_{X_{2}} \varphi F X_{1}, Y_{2}\right) \\
& +g\left(\left(\tilde{\nabla}_{X_{2}} \varphi\right) F X_{1}, Y_{2}\right)+\eta\left(X_{1}\right) g\left(X_{2}, Y_{2}\right) .
\end{aligned}
$$

Again, using (1.3) and (2.6), we derive

$$
\begin{aligned}
g\left(\tilde{\nabla}_{X_{2}} X_{1}, Y_{2}\right)= & T_{1} X_{1}(\ln f) g\left(X_{2}, T_{2} Y_{2}\right)+g\left(h\left(X_{2}, T_{1} X_{1}\right), F Y_{2}\right)-g\left(\tilde{\nabla}_{X_{2}} t F X_{1}, Y_{2}\right) \\
& -g\left(\tilde{\nabla}_{X_{2}} f F X_{1}, Y_{2}\right)+\eta\left(X_{1}\right) g\left(X_{2}, Y_{2}\right) .
\end{aligned}
$$


AJMS

27,1

8
Then, from (2.10) and (3.1), we obtain

$$
\begin{aligned}
g\left(\tilde{\nabla}_{X_{2}} X_{1}, Y_{2}\right) & =T_{1} X_{1}(\ln f) g\left(X_{2}, T_{2} Y_{2}\right)+g\left(h\left(X_{2}, T_{1} X_{1}\right), F Y_{2}\right) \\
& +\sin ^{2} \theta_{1} X_{1}(\ln f) g\left(X_{2}, Y_{2}\right) \\
& -\sin ^{2} \theta_{1} \eta\left(X_{1}\right) g\left(X_{2}, Y_{2}\right)+g\left(\tilde{\nabla}_{X_{2}} F T_{1} X_{1}, Y_{2}\right)+\eta\left(X_{1}\right) g\left(X_{2}, Y_{2}\right) \\
& =T_{1} X_{1}(\ln f) g\left(X_{2}, T_{2} Y_{2}\right)+g\left(h\left(X_{2}, T_{1} X_{1}\right), F Y_{2}\right) \\
& +\sin ^{2} \theta_{1} X_{1}(\ln f) g\left(X_{2}, Y_{2}\right) \\
& +\cos ^{2} \theta_{1} \eta\left(X_{1}\right) g\left(X_{2}, Y_{2}\right)-g\left(h\left(X_{2}, Y_{2}\right), F T_{1} X_{1}\right) .
\end{aligned}
$$

Thus, the result follows from (3.7) and (3.8), which proves the lemma completely.

The following useful relations are easily derived by interchanging $X_{1}$ by $T_{1} X_{1}, X_{2}$ by $T_{2} X_{2}$ and $Y_{2}$ by $T_{2} Y_{2}$ in Lemma 2.

$$
\begin{aligned}
& g\left(h\left(X_{2}, Y_{2}\right), F X_{1}\right)-g\left(h\left(X_{1}, X_{2}\right), F Y_{2}\right)= T_{1} X_{1}(\ln f) g\left(X_{2}, Y_{2}\right) \\
&-\left(X_{1}(\ln f)-\eta\left(X_{1}\right)\right) g\left(T_{2} X_{2}, Y_{2}\right), \\
& g\left(h\left(T_{2} X_{2}, Y_{2}\right), F T_{1} X_{1}\right)-g\left(h\left(T_{2} X_{2}, T_{1} X_{1}\right), F Y_{2}\right)= \cos ^{2} \theta_{2} T_{1} X_{1}(\ln f) g\left(X_{2}, Y_{2}\right) \\
&- \cos ^{2} \theta_{1}\left(X_{1}(\ln f)\right. \\
&-\left.\eta\left(X_{1}\right)\right) g\left(T_{2} X_{2}, Y_{2}\right), \\
& g\left(h\left(X_{2}, T_{2} Y_{2}\right), F T_{1} X_{1}\right)-g\left(h\left(X_{2}, T_{1} X_{1}\right), F T_{2} Y_{2}\right)=-\cos ^{2} \theta_{2} T_{1} X_{1}(\ln f) g\left(X_{2}, Y_{2}\right) \\
&+\cos ^{2} \theta_{1}\left(X_{1}(\ln f)\right. \\
&\left.-\eta\left(X_{1}\right)\right) g\left(T_{2} X_{2}, Y_{2}\right), \\
& g\left(h\left(T_{2} X_{2}, T_{2} Y_{2}\right), F T_{1} X_{1}\right)-g\left(h\left(T_{2} X_{2}, T_{1} X_{1}\right), F T_{2} Y_{2}\right)=-\cos ^{2} \theta_{2} T_{1} X_{1}(\ln f) \\
& g\left(T_{2} X_{2}, Y_{2}\right) \\
&- \cos ^{2} \theta_{1} \cos ^{2} \theta_{2}\left(X_{1}(\ln f)\right. \\
&-\left.\eta\left(X_{1}\right)\right) g\left(X_{2}, Y_{2}\right),
\end{aligned}
$$

$$
\begin{aligned}
g\left(h\left(T_{2} X_{2}, Y_{2}\right), F X_{1}\right)-g\left(h\left(T_{2} X_{2}, X_{1}\right), F Y_{2}\right) & =T_{1} X_{1}(\ln f) g\left(T_{2} X_{2}, Y_{2}\right) \\
& +\cos ^{2} \theta_{2}\left(X_{1}(\ln f)\right. \\
& \left.-\eta\left(X_{1}\right)\right) g\left(X_{2}, Y_{2}\right), \\
g\left(h\left(X_{2}, T_{2} Y_{2}\right), F X_{1}\right)-g\left(h\left(X_{2}, X_{1}\right), F T_{2} Y_{2}\right) & =T_{1} X_{1}(\ln f) g\left(X_{2}, T_{2} Y_{2}\right) \\
& -\cos ^{2} \theta_{2}\left(X_{1}(\ln f)\right. \\
& \left.-\eta\left(X_{1}\right)\right) g\left(X_{2}, Y_{2}\right)
\end{aligned}
$$

and

$g\left(h\left(T_{2} X_{2}, T_{2} Y_{2}\right), F X_{1}\right)-g\left(h\left(T_{2} X_{2}, X_{1}\right), F T_{2} Y_{2}\right)=\cos ^{2} \theta_{2} T_{1} X_{1}(\ln f) g\left(X_{2}, Y_{2}\right)$

$$
\begin{aligned}
- & \cos ^{2} \theta_{2}\left(X_{1}(\ln f)-\eta\left(X_{1}\right)\right) \\
& g\left(T_{2} X_{2}, Y_{2}\right) .
\end{aligned}
$$




\section{An inequality for warped product bi-slant submanifolds}

Let $M=M_{1} \times_{f} M_{2}$ be a warped product bi-slant submanifolds of a Kenmotsu manifold $\tilde{M}$; we decompose the normal bundle of $M$ as follows

$$
T^{\perp} M=F \mathfrak{D}_{1} \oplus F \mathfrak{D}_{2} \oplus \mu, \quad \mu \perp F \mathfrak{D}_{1} \oplus F \mathfrak{D}_{2},
$$

where $\mu$ is a $\varphi$-invariant normal subbundle of $T^{\perp} M$.

A warped product bi-slant submanifold $M=M_{1} \times{ }_{f} M_{2}$ of a Kenmotsu manifold $\tilde{M}$ is said to be mixed totally geodesic, if $h(X, Z)=0$, for any $X \in \mathfrak{D}_{1}$ and $Z \in \mathfrak{D}_{2}$, where $\mathfrak{D}_{1}$ and $\mathfrak{D}_{2}$ are the tangent bundles of $M_{1}$ and $M_{2}$, respectively.

Now, we set the following frame fields for an $n$-dimensional warped product bi-slant submanifold $M=M_{1} \times{ }_{f} M_{2}$ of a $(2 m+1)$-dimensional Kenmotsu manifold $\tilde{M}$ such that $\xi$ is tangent to $M_{1}$, where $M_{1}$ and $M_{2}$ are proper slant submanifolds of $\tilde{M}$ with slant angles $\theta_{1}$ and $\theta_{2}$ respectively. Let us consider the dimensions $\operatorname{dim}\left(M_{1}\right)=2 p+1$ and $\operatorname{dim} M_{2}=2 q$, i.e., $n=2 p+1+2 q$. Then the orthonormal frames of the corresponding tangent spaces $\mathfrak{D}_{1}$ and $\mathfrak{D}_{2}$, respectively are given by $\left\{e_{1}, \ldots, e_{p}, e_{p+1}=\sec \theta_{1} T_{1} e_{1}, \ldots, e_{2 p}=\sec \theta_{1} T_{1} e_{p}, e_{2 p+1}=\xi\right\}$ and $\left\{e_{2 p+2}=\bar{e}_{1}, \ldots, e_{2 p+1+q}=\bar{e}_{q}, e_{2 p+q+2}=\bar{e}_{q+1}=\sec \theta_{2} T_{2} \bar{e}_{1}, \ldots, e_{n}=\bar{e}_{2 q}=\sec \theta_{2} T_{2} \bar{e}_{q}\right\}$. Thus, the orthonormal frame fields of the normal subbundles of $F \mathfrak{D}_{1}, F \mathfrak{D}_{2}$ and $\mu$, respectively are $\left\{e_{n+1}=\tilde{e}_{1}=\csc \theta_{1} F e_{1}, \ldots, e_{n+p}=\tilde{e}_{p}=\csc \theta_{1} F e_{p}, e_{n+p+1}=\tilde{e}_{p+1}=\csc \theta_{1}\right.$ $\left.\sec \theta_{1} F T_{1} e_{1}, \ldots, e_{n+2 p}=\tilde{e}_{2 p}=\csc \theta_{1} \sec \theta_{1} F T_{1} e_{p}\right\},\left\{e_{n+2 p+1}=\tilde{e}_{2 p+1}=\widehat{e}_{1}=\csc \theta_{2} F \bar{e}_{1}, \ldots\right.$, $e_{n+2 p+q}=\tilde{e}_{2 p+q}=\widehat{e}_{q}=\csc \theta_{2} F \bar{e}_{q}, e_{n+2 p+q+1}=\tilde{e}_{2 p+q+1}=\widehat{e}_{q+1}=\csc \theta_{2} \sec \theta_{2} F T_{2} \bar{e}_{1}, \ldots$, $\left.e_{n+2 p+2 q}=\tilde{e}_{2 p+2 q}=\widehat{e}_{2 q}=\csc \theta_{2} \sec \theta_{2} F T_{2} \bar{e}_{q}\right\}$ and $\left\{e_{2 n}=\tilde{e}_{n}, \ldots, e_{2 m+1}=\tilde{e}_{2(m-n+1)}\right\}$.

Now, using the results of Section 3 and the above frame fields, we give following main result of this paper.

Theorem 4. Let $M=M_{1} \times{ }_{f} M_{2}$ be a mixed totally geodesic warped product bi-slant submanifold of a Kenmotsu manifold $\tilde{M}$ such that $\xi$ is tangent to $M_{1}$, where $M_{1}$ and $M_{2}$ are proper slant submanifolds of $\tilde{M}$ with slant angles $\theta_{1}$ and $\theta_{2}$, respectively. Then

(i) The second fundamental form hof $M$ satisfies the following inequality

$$
\|h\|^{2} \geq 2 q \csc ^{2} \theta_{1}\left(\cos ^{2} \theta_{1}+\cos ^{2} \theta_{2}\right)\left(\|\vec{\nabla}(\ln f)\|^{2}-1\right),
$$

where $2 q=\operatorname{dim} M_{2}$ and $\vec{\nabla}(\ln f)$ is the gradient of $\ln f$ along $M_{1}$.

(ii) If equality sign in (i) holds identically, then:

(a) $M_{1}$ is a totally geodesic submanifold of $\tilde{M}$;

(b) $M_{2}$ is a totally umbilical submanifold of $\tilde{M}$.

Proof. From (2.4), we have

$$
\|h\|^{2}=\sum_{i, j=1}^{n} g\left(h\left(e_{i}, e_{j}\right), h\left(e_{i}, e_{j}\right)\right)=\sum_{r=n+1}^{2 m+1} \sum_{i, j=1}^{n} g\left(h\left(e_{i}, e_{j}\right), e_{r}\right)^{2} .
$$

Splitting the above expression for the tangent bundles of $M_{1}$ and $M_{2}$, we derive

$$
\begin{aligned}
\|h\|^{2}= & \sum_{r=1}^{2 m+1} \sum_{i, j=1}^{2 p+1} g\left(h\left(e_{i}, e_{j}\right), e_{r}\right)^{2}+2 \sum_{r=1}^{2 m+1} \sum_{i=1}^{2 p+1} \sum_{j=1}^{2 q} g\left(h\left(e_{i}, \bar{e}_{j}\right), e_{r}\right)^{2} \\
& +\sum_{r=1}^{2 m+1} \sum_{i, j=1}^{2 q} g\left(h\left(\bar{e}_{i}, \bar{e}_{j}\right), e_{r}\right)^{2} .
\end{aligned}
$$

Submanifolds of Kenmotsu manifolds 
AJMS

27,1

10

Since $M$ is mixed totally geodesic, then the second term in the right hand side of (4.3) is identically zero. Thus, we find

$$
\begin{aligned}
\|h\|^{2} & =\sum_{r=1}^{2 p} \sum_{i, j=1}^{2 p+1} g\left(h\left(e_{i}, e_{j}\right), \tilde{e}_{r}\right)^{2}+\sum_{r=1}^{2 q} \sum_{i, j=1}^{2 p+1} g\left(h\left(e_{i}, e_{j}\right), \widehat{e}_{r}\right)^{2} \\
& +\sum_{r=n}^{2(m-n+1)} \sum_{i, j=1}^{2 p+1} g\left(h\left(e_{i}, e_{j}\right), \tilde{e}_{r}\right)^{2} \\
& +\sum_{r=1}^{2 p} \sum_{i, j=1}^{2 q} g\left(h\left(\bar{e}_{i}, \bar{e}_{j}\right), \tilde{e}_{r}\right)^{2}+\sum_{r=1}^{2 q} \sum_{i, j=1}^{2 q} g\left(h\left(\bar{e}_{i}, \bar{e}_{j}\right), \widehat{e}_{r}\right)^{2} \\
& +\sum_{r=n}^{2(m-n+1)} \sum_{i, j=1}^{2 q} g\left(h\left(\bar{e}_{i}, \bar{e}_{j}\right), \tilde{e}_{r}\right)^{2} .
\end{aligned}
$$

The third and sixth terms have $\mu$-components and we could not find any relation for warped products in terms of $\mu$-components, therefore we shall leave these two terms. Also, we could not find any relations for $g\left(h\left(e_{i}, e_{j}\right), \tilde{e}_{r}\right), i, j=1, \ldots, 2 p+1, r=1, \ldots, 2 p$ and $g\left(h\left(\bar{e}_{i}, \bar{e}_{j}\right)\right.$, $\left.\widehat{e}_{r}\right), i, j, r=1, \ldots, 2 q$. Therefore, we also leave the first and fifth terms. By using Lemma 1(ii) for a mixed totally geodesic warped product the second term in the right hand side is also zero. Thus, the evaluated term is only fourth term which can be expressed by using the constructed frame fields as follows

$$
\begin{aligned}
\|h\|^{2} \geq & \csc ^{2} \theta_{1} \sec ^{2} \theta_{2} \sum_{r=1}^{p} \sum_{i, j=1}^{q} g\left(h\left(\bar{e}_{i}, T_{2} \bar{e}_{j}\right), F e_{r}\right)^{2} \\
& +\csc ^{2} \theta_{1} \sec ^{2} \theta_{2} \sum_{r=1}^{p} \sum_{i, j=1}^{q} g\left(h\left(T_{2} \bar{e}_{i}, \bar{e}_{j}\right), F e_{r}\right)^{2} \\
& +\csc ^{2} \theta_{1} \sec ^{2} \theta_{1} \sec ^{2} \theta_{2} \sum_{r=1}^{p} \sum_{i, j=1}^{q} g\left(h\left(\bar{e}_{i}, T_{2} \bar{e}_{j}\right), F T_{1} e_{r}\right)^{2} \\
& +\csc ^{2} \theta_{1} \sec ^{2} \theta_{1} \sec ^{2} \theta_{2} \sum_{r=1}^{p} \sum_{i, j=1}^{q} g\left(h\left(T_{2} \bar{e}_{i}, \bar{e}_{j}\right), F T_{1} e_{r}\right)^{2} \\
& +\csc ^{2} \theta_{1} \sum_{r=1}^{p} \sum_{i, j=1}^{q} g\left(h\left(\bar{e}_{i}, \bar{e}_{j}\right), F e_{r}\right)^{2} \\
& +\csc ^{2} \theta_{1} \sec ^{2} \theta_{1} \sum_{r=1}^{p} \sum_{i, j=1}^{q} g\left(h\left(\bar{e}_{i}, \bar{e}_{j}\right), F T_{1} e_{r}\right)^{2} \\
& +\csc ^{2} \theta_{1} \sec ^{4} \theta_{2} \sum_{r=1}^{p} \sum_{i, j=1}^{q} g\left(h\left(T_{2} \bar{e}_{i}, T_{2} \bar{e}_{j}\right), F e_{r}\right)^{2} \\
& +\csc ^{2} \theta_{1} \sec ^{2} \theta_{1} \sec ^{4} \theta_{2} \sum_{r=1}^{p} \sum_{i, j=1}^{q} g\left(h\left(T_{2} \bar{e}_{i}, T_{2} \bar{e}_{j}\right), F T_{1} e_{r}\right)^{2} .
\end{aligned}
$$

Using Lemma 2 and the relations (3.9)-(3.15) for a mixed totally geodesic warped product submanifold, we derive 


$$
\begin{aligned}
\|h\|^{2} & \geq 2 q \csc ^{2} \theta_{1} \cos ^{2} \theta_{2} \sum_{r=1}^{p}\left(e_{r}(\ln f)-\eta\left(e_{r}\right)\right)^{2} \\
& +2 q \csc ^{2} \theta_{1} \sec ^{2} \theta_{1} \cos ^{2} \theta_{2} \sum_{r=1}^{p}\left(T_{1} e_{r}(\ln f)\right)^{2} \\
& +2 q \csc ^{2} \theta_{1} \sum_{r=1}^{p}\left(T_{1} e_{r}(\ln f)\right)^{2}+2 q \csc ^{2} \theta_{1} \cos ^{2} \theta_{1} \sum_{r=1}^{p}\left(e_{r}(\ln f)-\eta\left(e_{r}\right)\right)^{2} \\
& =2 q \csc ^{2} \theta_{1}\left(\cos ^{2} \theta_{1}+\cos ^{2} \theta_{2}\right) \sum_{r=1}^{p}\left(e_{r}(\ln f)-\eta\left(e_{r}\right)\right)^{2} \\
& +2 q \csc ^{2} \theta_{1}\left(1+\cos ^{2} \theta_{2} \sec ^{2} \theta_{1}\right) \sum_{r=1}^{p}\left(T_{1} e_{r}(\ln f)\right)^{2} .
\end{aligned}
$$

Since $\eta\left(e_{r}\right)=0, r=1, \ldots, 2 p$, then above expression will be

$$
\begin{aligned}
\|h\|^{2} & \geq 2 q \csc ^{2} \theta_{1}\left(\cos ^{2} \theta_{1}+\cos ^{2} \theta_{2}\right) \sum_{r=1}^{p}\left(e_{r}(\ln f)\right)^{2} \\
& +2 q \csc ^{2} \theta_{1}\left(1+\cos ^{2} \theta_{2} \sec ^{2} \theta_{1}\right) \sum_{r=1}^{2 p+1}\left(T_{1} e_{r}(\ln f)\right)^{2} \\
& -2 q \csc ^{2} \theta_{1}\left(1+\cos ^{2} \theta_{2} \sec ^{2} \theta_{1}\right) \sum_{r=p+1}^{2 p} g\left(e_{r}, T_{1} \vec{\nabla}(\ln f)\right)^{2} \\
& -2 q \csc ^{2} \theta_{1}\left(1+\cos ^{2} \theta_{2} \sec ^{2} \theta_{1}\right)\left(T_{1} e_{2 p+1}(\ln f)\right)^{2} .
\end{aligned}
$$

Since $T_{1} e_{2 p+1}=T_{1} \xi=0$, (4.7) can be written as

$$
\begin{aligned}
\|h\|^{2} & \geq 2 q \csc ^{2} \theta_{1}\left(\cos ^{2} \theta_{1}+\cos ^{2} \theta_{2}\right) \sum_{r=1}^{p}\left(e_{r}(\ln f)\right)^{2} \\
& +2 q \csc ^{2} \theta_{1}\left(1+\cos ^{2} \theta_{2} \sec ^{2} \theta_{1}\right)\left\|T_{1} \vec{\nabla}(\ln f)\right\|^{2} \\
& -2 q \csc ^{2} \theta_{1} \sec ^{2} \theta_{1}\left(1+\cos ^{2} \theta_{2} \sec ^{2} \theta_{1}\right) \sum_{r=1}^{p} g\left(T_{1} e_{r}, T_{1} \vec{\nabla}(\ln f)\right)^{2} .
\end{aligned}
$$

Now, we compute the second term as follows

$$
\left\|T_{1} \vec{\nabla}(\ln f)\right\|^{2}=g\left(T_{1} \vec{\nabla}(\ln f), T_{1} \vec{\nabla}(\ln f)\right) .
$$

Using (2.8), we get

$$
\left\|T_{1} \vec{\nabla}(\ln f)\right\|^{2}=\cos ^{2} \theta_{1}\left(g(\vec{\nabla}(\ln f), \vec{\nabla}(\ln f))-(\eta(\vec{\nabla} \ln f))^{2}\right) .
$$

Using the gradient definition and Lemma 1(i), we get

$$
\left\|T_{1} \vec{\nabla}(\ln f)\right\|^{2}=\cos ^{2} \theta_{1}\left(\|\vec{\nabla}(\ln f)\|^{2}-1\right) .
$$


AJMS

27,1

12

Then from (4.8) and (4.9), we derive

$$
\begin{aligned}
\|h\|^{2} & \geq 2 q \csc ^{2} \theta_{1}\left(\cos ^{2} \theta_{1}+\cos ^{2} \theta_{2}\right) \sum_{r=1}^{p}\left(e_{r}(\ln f)\right)^{2} \\
& +2 q \csc ^{2} \theta_{1}\left(\cos ^{2} \theta_{1}+\cos ^{2} \theta_{2}\right)\left(\|\vec{\nabla}(\ln f)\|^{2}-1\right) \\
& -2 q \csc ^{2} \theta_{1}\left(1+\cos ^{2} \theta_{2} \sec ^{2} \theta_{1}\right) \csc ^{2} \theta_{1} \sum_{r=1}^{p} g\left(e_{r}, \vec{\nabla}(\ln f)\right)^{2} \\
& =2 q \csc ^{2} \theta_{1}\left(\cos ^{2} \theta_{1}+\cos ^{2} \theta_{2}\right) \sum_{r=1}^{p}\left(e_{r}(\ln f)\right)^{2} \\
& +2 q \csc ^{2} \theta_{1}\left(\cos ^{2} \theta_{1}+\cos ^{2} \theta_{2}\right)\left(\|\vec{\nabla}(\ln f)\|^{2}-1\right) \\
& -2 q \csc ^{2} \theta_{1}\left(\cos ^{2} \theta_{1}+\cos ^{2} \theta_{2}\right) \sum_{r=1}^{p}\left(e_{r}(\ln f)\right)^{2} \\
& =2 q \csc ^{2} \theta_{1}\left(\cos ^{2} \theta_{1}+\cos ^{2} \theta_{2}\right)\left(\|\vec{\nabla}(\ln f)\|^{2}-1\right)
\end{aligned}
$$

which is inequality (4.2). For the equality case, since $M$ is mixed totally geodesic, then

$$
h\left(\mathfrak{D}_{1}, \mathfrak{D}_{2}\right)=\{0\} .
$$

From the leaving third and sixth terms in the right hand side of (4.4), we have

$$
h(X, Y) \perp \mu,
$$

for any $X, Y \in T M$. Also, from the leaving first term of (4.4), we find

$$
h\left(\mathfrak{D}_{1}, \mathfrak{D}_{1}\right) \perp F \mathfrak{D}_{1} .
$$

Then from (4.11) and (4.12), we conclude that

$$
h\left(\mathfrak{D}_{1}, \mathfrak{D}_{1}\right) \subset F \mathfrak{D}_{2} .
$$

But for a mixed totally geodesic submanifold, from Lemma 1(ii), we get

$$
h\left(\mathfrak{D}_{1}, \mathfrak{D}_{1}\right) \perp F \mathfrak{D}_{2} .
$$

Thus, from (4.13) and (4.14), we find

$$
h\left(\mathfrak{D}_{1}, \mathfrak{D}_{1}\right)=\{0\} .
$$

Since $M_{1}$ is totally geodesic in $M[5,11]$, then using this fact with (4.10) and (4.15) we conclude that $M_{1}$ is a totally geodesic submanifold of $\tilde{M}$, which is the first relation of inequality. Similarly, from the leaving fifth term in the right hand side of (4.4), we get

$$
h\left(\mathfrak{D}_{2}, \mathfrak{D}_{2}\right) \perp F \mathfrak{D}_{2} \text {. }
$$

Thus, (4.11) and (4.16) yield

$$
h\left(\mathfrak{D}_{2}, \mathfrak{D}_{2}\right) \subset F \mathfrak{D}_{1} .
$$

Since $M_{2}$ is totally umbilical in $M$ [5,11], using this fact with (4.10) and (4.17), we conclude that $M_{2}$ is a totally umbilical submanifold of $\tilde{M}$, which proves the statement $(b)$. Hence, the proof is complete. 
5. Some applications of derived inequality

In this section, we give some applications of the derived inequality (4.2).

1. In Theorem 4, if $\theta_{1}=\frac{\pi}{2}$ and $\theta_{2}=0$, then the warped product bi-slant $M_{1} \times_{f} M_{2}$ takes the form $M_{\perp} \times{ }_{f} M_{T}$ i.e., $M$ is a contact CR-warped product studied in [27]. In this case, the inequality (4.2) will be $\|h\|^{2} \geq 2 q\left(\|\vec{\nabla}(\ln f)\|^{2}-1\right)$, which is the main Theorem 3.4 of [27].

2. If we consider $\theta_{1}=\theta$ and $\theta_{2}=0$ in a warped product bi-slant submanifold $M=M_{1} \times{ }_{f} M_{2}$, then $M$ is a warped product semi-slant submanifold of the form $M_{\theta} \times{ }_{f} M_{T}$ studied in [30]. Then, the inequality (4.2) change into $\|h\|^{2} \geq 2 q\left(\csc ^{2} \theta+\right.$ $\left.\cot ^{2} \theta\right)\left(\|\vec{\nabla}(\ln f)\|^{2}-1\right)$, which is Theorem 4.2 of [30]. Thus, Theorem 4.2 of [30] is a special case of Theorem 4 .

3. Also, if we consider $\theta_{1}=\theta$ and $\theta_{2}=\frac{\pi}{2}$ in a warped product bi-slant submanifold $M=M_{1} \times{ }_{f} M_{2}$ of a Kenmotsu manifold $\tilde{M}$, then $M$ turns into a warped product pseudoslant submanifold of the form $M=M_{\theta} \times{ }_{f} M_{\perp}$, where $M_{\theta}$ and $M_{\perp}$ are proper slant and antiinvariant submanifolds of $\tilde{M}$, respectively. In this case, if we put $\theta_{1}=\theta$ and $\theta_{2}=\frac{\pi}{2}$ in Theorem 4, then inequality (4.2) will be $\|h\|^{2} \geq 2 q \cot ^{2} \theta\left(\|\vec{\nabla}(\ln f)\|^{2}-1\right)$, which the inequality (5.1) of [1]. Hence, Theorem 5.1 of [1] is a special case of Theorem 4.

4. Similarly, if we assume that $\theta_{1}=\frac{\pi}{2}$ and $\theta_{2}=\theta$ in a warped product bi-slant submanifold $M=M_{1} \times{ }_{f} M_{2}$ of a Kenmotsu manifold $\tilde{M}$, then $M$ is a warped product pseudo-slant submanifold $M=M_{\perp} \times{ }_{f} M_{\theta}$ such that $M_{\perp}$ is an anti-invariant submanifold and $M_{\theta}$ is a proper slant submanifold of $\tilde{M}$. Thus inequality (4.2) takes the form $\|h\|^{2} \geq 2 q \cos ^{2} \theta\left(\|\vec{\nabla}(\ln f)\|^{2}-1\right)$, which the inequality (4.1) of [23]. Hence, Theorem 4.1 of [23] is a special case of Theorem 4.

\section{References}

[1] F.R. Al-Solamy, M.F. Naghi, S. Uddin, Geometry of warped product pseudo-slant submanifolds of Kenmotsu manifolds, Quaest. Math. 42 (3) (2019) 373-389, http://dx.doi.org/10.2989/16073606. 2018.1452800.

[2] L.S. Alqahtani, M.S. Stankovic, S. Uddin, Warped product bi-slant submanifolds of cosymplectic manifolds, Filomat 31 (16) (2017) 5065-5071, http://dx.doi.org/10.2298/FIL1716065A.

[3] K. Arslan, R. Ezentas, I. Mihai, C. Murathan, Contact CR-warped product submanifolds in Kenmotsu space forms, J. Korean Math. Soc. 42 (2005) 1101-1110.

[4] M. Atceken, Warped product semi-slant submanifolds in Kenmotsu manifolds, Turk. J. Math. 36 (2012) 319-330.

[5] R.L. Bishop, B. O’Neill, Manifolds of negative curvature, Trans. Amer. Math. Soc. 145 (1969) 1-49.

[6] D.E. Blair, Contact Manifolds in Riemannian Geometry, in Lecture Notes in Mathematics, vol. 509, Springer-Verlag, New York, 1976.

[7] J.L. Cabrerizo, A. Carriazo, L.M. Fernandez, M. Fernandez, Semi-slant submanifolds of a Sasakian manifold, Geom. Dedicata 78 (2) (1999) 183-199.

[8] J.L. Cabrerizo, A. Carriazo, L.M. Fernandez, M. Fernandez, Slant submanifolds in Sasakian manifolds, Glasg. Math. J. 42 (2000) 125-138.

[9] B.-Y. Chen, Geometry of Slant Submanifolds, Katholieke Universiteit Leuven, 1990.

[10] B.-Y. Chen, Slant immersions, Bull. Aust. Math. Soc. 41 (1990) 135-147.

[11] B.-Y. Chen, Geometry of warped product CR-submanifolds in Kaehler manifolds, Monatsh. Math. 133 (2001) 177-195. 
AJMS

27,1

14

[12] B.-Y. Chen, Geometry of warped product CR-submanifolds in Kaehler manifolds II, Monatsh. Math. 134 (2001) 103-119.

[13] B.-Y. Chen, Pseudo-Riemannian Geometry $\delta$-Invariants and Applications, World Scientific, Hackensack, NJ, 2011.

[14] B.-Y. Chen, Geometry of warped product submanifolds: a survey, J. Adv. Math. Stud. 6 (2) (2013) $1-43$.

[15] B.-Y. Chen, Differential Geometry of Warped Product Manifolds and Submanifolds, World Scientific, Hackensack, NJ, 2017.

[16] B.-Y. Chen, S. Uddin, Warped product pointwise bi-slant submanifolds of Kaehler manifolds, Publ. Math. Debr. 92 (1-2) (2018) 183-199.

[17] I. Hasegawa, I. Mihai, Contact CR-warped product submanifolds in Sasakian manifolds, Geom. Dedicata 102 (2003) 143-150.

[18] K. Kenmotsu, A class of almost contact Riemannian manifolds, Tohoku Math. J. 24 (1972) 93-103.

[19] K. Matsumoto, I. Mihai, Warped product submanifolds in Sasakian space forms, SUT J. Math. 38 (2) (2002) 135-144.

[20] A. Mihai, Warped product submanifolds in complex space forms, Acta Sci. Math. Szeged 70 (2004) 419-427.

[21] A. Mihai, Warped product submanifolds in quaternion space forms, Rev. Roum. Math. Pures Appl. 50 (2005) 283-291.

[22] C. Murathan, K. Arslan, R. Ezentas, I. Mihai, Warped product submanifolds in Kenmotsu space forms, Taiwanese J. Math. 10 (6) (2006) 1431-1441.

[23] M.F. Naghi, S. Uddin, F.R. Al-Solamy, Warped product submanifolds of Kenmotsu manifolds with slant fiber, Filomat 32 (6) (2018) 2115-2126.

[24] B. Sahin, Warped product pointwise semi-slant submanifolds of Kaehler manifolds, Port. Math. 70 (2013) 252-268.

[25] S. Uddin, Geometry of warped product semi-slant submanifolds of Kenmotsu manifolds, Bull. Math. Sci. 8 (2018) 435-451, http://dx.doi.org/10.1007/s13373-017-0106-9.

[26] S. Uddin, F.R. Al-Solamy, Warped product pseudo-slant immersions in Sasakian manifolds, Publ. Math. Debr. 91 (3-4) (2017) 331-348, http://dx.doi.org/10.5486/PMD.2017.7640.

[27] S. Uddin, A. Alghanemi, M.F. Naghi, F.R. Al-Solamy, Another class of warped product CRsubmanifolds in Kenmotsu manifolds, J. Math. Comput. Sci. 17 (2017) 148-157.

[28] S. Uddin, B.-Y. Chen, F.R. Al-Solamy, Warped product bi-slant immersions in Kaehler manifolds, Mediterr. J. Math. 14 (2) (2017) http://dx.doi.org/10.1007/s00009-017-0896-8, Art. 95, p. 11.

[29] S. Uddin, V.A. Khan, K.A. Khan, Warped product submanifolds of a Kenmotsu manifold, Turk. J. Math. 36 (2012) 319-330.

[30] S. Uddin, M.F. Naghi, F.R. Al-Solamy, Another class of warped product submanifolds of Kenmotsu manifolds, RACSAM 112 (4) (2018) 1141-1155, http://dx.doi.org/10.1007/s13398-017-0415-6.

[31] K. Yano, M. Kon, Structures on Manifolds, in: Series in Pure Mathematics, World Scientific Publishing Co., Singapore, 1984.

\section{Corresponding author}

Siraj Uddin can be contacted at: siraj.ch@gmail.com

For instructions on how to order reprints of this article, please visit our website:

www.emeraldgrouppublishing.com/licensing/reprints.htm

Or contact us for further details: permissions@emeraldinsight.com 